\title{
Attention functions in children with spina bifida with and without hydrocephalus
}

\author{
Anja Vinck*, Jan Rotteveel, Reinier Mullaart and Ben Maassen
}

\author{
Address: Interdisciplinary Child Neurology Centre 945, University Medical Centre St Radboud P.O. Box 9101, 6500 HB Nijmegen, The \\ Netherlands \\ Email: Anja Vinck* - a.vinck@cukz.umcn.nl \\ * Corresponding author
}

from 49th Annual Meeting of the Society for Research into Hydrocephalus and Spina Bifida

Barcelona, Spain, 29 June - 2 July 2005

Published: 30 December 2005

Cerebrospinal Fluid Research 2005, 2(Suppl I):S39 doi:10.1 I86/I743-8454-2-SI-S39

\section{Background}

This study is part of the multidisciplinary research program "Prognosis of Spina Bifida" of the Radboud University Nijmegen (The Netherlands). Recent studies indicate that children with hydrocephalus have difficulty on attention and memory tests. However, it remains unclear whether these problems stem from deficits in the attentional system or from the perceptual and motor requirements of the task. The purpose of the current study is to investigate attention functions in children with spina bifida and hydrocephalus (SBH) as compared to those without hydrocephalus (SB) by administering a neuropsychological test procedure that takes into account the different task demands.

\section{Materials and methods}

55 children between 6 and 15 years of age were recruited from the spina bifida team of the Radboud University Nijmegen, Medical Centre. All children underwent a neuropsychological assessment which consisted of tests on a wide range of cognitive functions, comprising speed of information processing, selective and sustained attention, simultaneous and sequential memory. Of the whole group of children, 36 were included for further analysis on the criteria documented presence or absence of hydrocephalus and total IQ at least 70. By comparing computerized reaction time tasks with paper-and-pencil tasks we can distinguish between attentional problems due to impaired motor speed or due to attentional deficits.

\section{Results}

Results reveal that hydrocephalic children are impaired on certain paper-and-pencil attention subtests of the WISCIII and the Bourdon-Vos Concentration Test compared to normative data of healthy controls. SBH children show deficits concerning the 'freedom from distractability factor' of the WISC-III in contrast to children without hydrocephalus. However, there were no differences between SBH and SB concerning computerized attention tasks (sutained and focused attention, impulsivity), and the Stroop-Color-Word Task (distractability).

\section{Conclusion}

When motor demands are minimized, children with hydrocephalus do not display attention problems. From this, we conclude that SBH children are not impaired in attention in general, but perform poorer on attentional tasks due to the motor demands inherent to the tasks. Therefore, it is important to assess attention in SBH with computerized simple reaction time tasks, which measure the different aspects of attention. More research is needed among individuals with SBH before the use of computerized diagnostic as a stand-alone tool apart from adjunctive cognitive assessment instruments is valid. 